\title{
Améliorations des caractéristiques de la présure fixée sur verre poreux
}

\author{
par \\ M. PAQUOT, Ph. THONART et C. DEROANNE \\ Faculté des Sciences Agronomiques \\ Chaire de Technologie Agricole et Alimentaire \\ 5800 Gembloux (Belgique)
}

\section{I. - INTRODUCTION}

L'énorme marché potentiel des enzymes a conduit à une recherche de leurs meilleures conditions d'exploitation. Dans cette optique, les enzymes immobilisées suscitent, depuis quelques-années, un immense intérêt, tant théorique que technologique.

La fixation d'enzyme sur support insoluble présente de nombreux avantages, notamment l'utilisation en continu des enzymes et l'obtention d'un produit final non contaminé par une protéine étrangère $[13,8,4]$.

Les industries alimentaires, grandes utilisatrices d'enzymes, s'intéressent fortement à cette technique. Parmi ces industries, la laiterie constitue un bon exemple de la possibilité d'utilisation avantageuse d'enzyme fixée. Suite à la pénurie de présure animale, l'utilisation de succédanés d'origine microbienne est envisagée [6]. Or, ces succédanés ne donnent pas toujours satisfaction car ils provoquent des modifications de caractère organoleptique. Les espérances mises en l'utilisation repétée du même enzyme, fixée sur support inerte, pour parer à cette pénurie sont dès lors très grandes. Malheureusement l'activité résiduelle de la présure fixée est faible $[1,5]$; en conséquence, son utilisation sous une forme immobilisée n'est pas encore économiquement rentable.

Afin d'accroître les possibilités d'utilisation de la présure fixée, nous nous proposons d'améliorer l'activité résiduelle et la stabilité au stockage de celle-ci en choisissant comme support des billes de verre poreux et comme réaction de couplage, la réaction de diazotation. 


\section{II. - MATERIEL ET METHODES}

\subsection{Réactifs}

Bio glass : billes de verre poreux de dimensions 50 à 100 mesh. (Bio-Rad Laboratories); $\gamma$-aminopropyltriéthoxysilane: (Aldrich Chemical) ; Chlorure de p-nitrobenzoyle (Merck); triéthylamine (Merck); Dithionite de sodium Merck): Tampon phosphate de sodium $(0,01 \mathbf{M}$ ou $0,05 \mathrm{M})$ de $\mathrm{pH} 6,2$ et $\mathrm{pH}$ 7. Tampon acétate de $\mathrm{Na} 0,01 \mathrm{M}$ de pH $4,1-4,8$ et 5,4 .

\subsection{Méthodes : mesure de l'activité coagulante}

Le dosage est basé sur la mesure du temps de coagulation du lait, suite à l'action de la présure. Le lait est préparé à partir de poudre de lait écrémé à la concentration de $8 \mathrm{p} .100$. Nous avons utilisé le mode opératoire décrit par Houins et al. [6].

La figure 1 montre la linéarité du dosage en fonction de la concentration enzymatique.

Unité de force : activité requise pour coaguler $10 \mathrm{ml}$ de lait écrémé ( 8 p. 100 de MS) en $1000 \mathrm{mn}$ en ajoutant $1 \mathrm{ml}$ de la solution (suspension) enzymatique dans les conditions opératoires définies ( $\mathrm{t}^{\circ}=35^{\circ} \mathrm{C}$, concentration en $\mathrm{CaCl}_{2}=0,01 \mathrm{M}$ ) ce qui correspond à $6320.10^{-8}$ unités R.U.

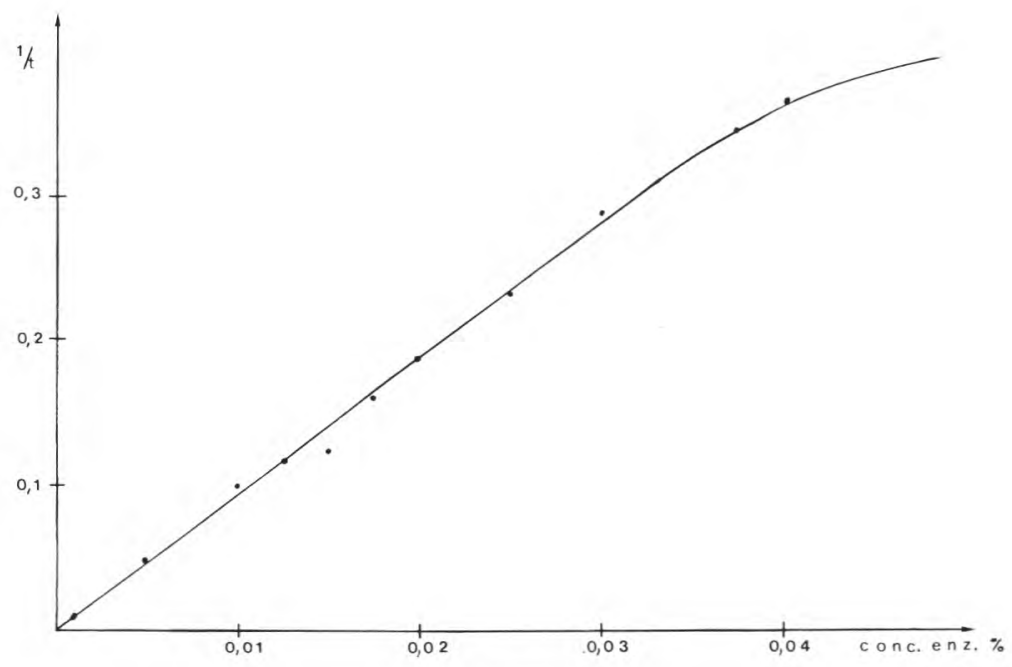

fig. 1

Présure animale : relation entre l'inverse du temps de coagulation (en minutes) et la concentration enzymatique. 


\subsection{Fixation de l'enzyme sur billes de verre silanées $[11,7,12]$}

Les billes de verre sont agitées pendant $16 \mathrm{~h}$ dans $10 \mathrm{ml}$ de toluène contenant 10 p. 100 de $\gamma$-aminopropyltriéthoxysilane.

Les billes silanées réagissent durant $1 \mathrm{~h}$ avec le chlorure de p-nitrobenzoyle dans $10 \mathrm{ml}$ de chloroforme contenant 5 p. 100 de triéthylamine [12], elles sont récupérées par filtration.

La forme arylnitrée est convertie en arylaminée par l'addition de $10 \mathrm{ml}$ d'une solution de $\mathrm{Na}_{2} \mathrm{~S}_{2} \mathrm{O}_{4}$ à $4 \mathrm{p} .100$ pendant $1 \mathrm{~h}$.

La forme arylaminée obtenue est agitée dans $10 \mathrm{ml}$ de $\mathrm{HCl} 0,66 \mathrm{M}$ et l'addition de dix fois $0,2 \mathrm{ml}$ de $\mathrm{NaNO}_{2}$ à 20 p. 100 (à intervalles de $90 \mathrm{~s}$ ) permet la formation du chlorure de diazonium.

Les billes sont filtrées puis lavées avec $\mathrm{HCl} 1 \mathrm{mM}$. Elles sont alors transférées dans un milieu de couplage approprié à l'enzyme soit dans $20 \mathrm{ml}$ d'eau distillée contenant $0,1 \mathrm{~g}$ de présure commerciale (soit environ $10 \mathrm{mg}$ de protéine) pour $1 \mathrm{~g}$ de billes de verre.

Le couplage a lieu pendant $4 \mathrm{~h}$ sous agitation. Les billes de verre sont ensuite lavées trois fois avec $20 \mathrm{ml}$ d'eau distillée et conservées à $4^{\circ} \mathrm{C}$ dans le même milieu.

\subsection{Fixation de l'enzyme sur billes de verre par l'intermédiaire de la glutaraldéhyde}

Après silanisation comme décrit précédemment, les billes sont traitées $30 \mathrm{mn}$ dans $20 \mathrm{ml}$ d'une solution aqueuse à 1 p. 100 de glutaraldéhyde. Après $30 \mathrm{mn}$, l'enzyme est ajoutée comme décrit précédemment.

\subsection{Protection par le sérum albumine}

Après diazotation et couplage de l'enzyme pendant $4 \mathrm{~h}, 20 \mathrm{mg}$ de sérum albumine sont ajoutés et agités pendant $5 \mathrm{~h}$. Les billes de verre sont ensuite lavées et stockées à $4^{\circ} \mathrm{C}$.

\subsection{Action de la glutaraldéhyde après diazotation}

Après la fixation de la présure par diazotation suivant la méthode décrite ci-dessus, 2,2 $\mathrm{ml}$ de glutaraldéhyde à $1 \mathrm{p} .100$ sont ajoutées pendant $18 \mathrm{~h}$. Les billes de verre sont ensuite lavées et stockées à $4^{\circ} \mathrm{C}$.

\section{III. - RESULTATS}

\subsection{Fixation de la présure}

Par la méthode de fixation utilisant la diazotation sur billes de verre silanisées $[11,7,12]$, nous avons pu immobiliser la présure 
L'activité résiduelle est en moyenne de 11,7 p. 100 et le rendement de fixation est de 96,5 p.100. Malheureusement, l'activité de l'enzyme fixée dans un tampon acétate de $\mathrm{pH}$ 4,8 décroît rapidement. Après $13 \mathrm{j}$ de conservation, 81,6 p. 100 de l'activité sont perdus.

\subsection{Amélioration de l'activité résiduelle}

L'amélioration de l'activité résiduelle a été étudiée à trois niveaux :

1) Etude de la préparation des billes de verre.

2) Etude du couplage.

3) Effet protecteur.

1) Etude DE LA PRÉPARATION DES BILLES DE VERRE

a) Après silanisation, les billes sont séchées à $110^{\circ} \mathrm{C}$ durant $12 \mathrm{~h}$ pour renforcer l'adhérence du dérivé silané. Les opérations suivantes ont été décrites dans les modes opératoires. Aucune amélioration de l'activité résiduelle n'a pu être décelée.

b) Pour étudier l'influence de la liaison entre le verre silané et l'enzyme, la diazotation a été remplacée par un réactif bifonctionnel, la glutaraldéhyde. Des résultats analogues à la méthode classique ont été enregistrés.

2) Etude du COUPLAGE

Nous avons testé différentes durées de couplage ainsi que l'importance de ce facteur sur l'activité résiduelle et le rendement de fixation. La figure 2 reprend les résultats. L'activité résiduelle est indépendante de la durée du couplage, alors que le rendement de

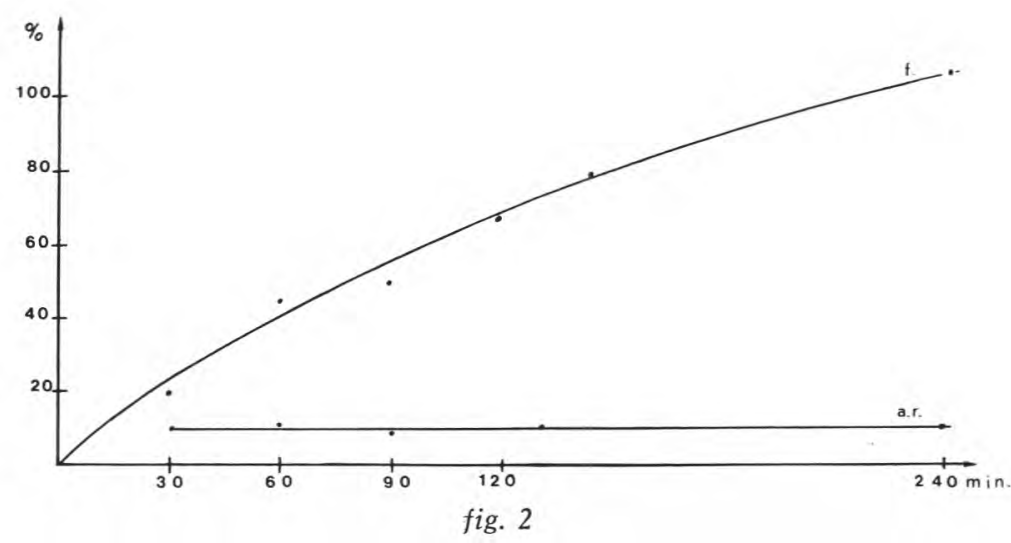

Etude de l'influence du temps de couplage sur le rendement de la fixation (f) et l'activité résiduelle (a.r.) de l'enzyme fixée. 
fixation s'accroît progressivement pour atteindre 96,5 p. 100 après $240 \mathrm{mn}$.

- Le pH et la force ionique du milieu de couplage peuvent influencer l'activité résiduelle et le rendement de fixation. Les résultats obtenus avec différents milieux de couplage sont repris au tableau 1. Aucune amélioration sensible n'a été enregistrée.

\section{TABLEAU 1}

Influence du milieu de couplage sur l'activité résiduelle et sur le rendement. Le rendement est la quantité de protéine effectivement fixée comparativement à la quantité de protéine mise en œuvre exprimée en p.100. L'activité résiduelle est l'activité coagulante des protéines erfectivement fixée comparativement à l'activité d'une même quantité d'enzyme libre exprimée en p. 100.

\begin{tabular}{l|r|r}
\multicolumn{1}{c|}{ Milieux de couplages } & $\begin{array}{r}\text { Activité } \\
\text { résiduelle }\end{array}$ & Rendement \\
\cline { 2 - 3 } & 14,9 p. 100 & 98,8 p. 100 \\
Tampon phosphate pH 6,2 0,01 M & 8,9 p. 100 & 95,7 p. 100 \\
Tampon phosphate pH 6,2 0,05 M & 13,7 p. 100 & 96,8 p. 100 \\
Tampon phosphate pH 7 0,01 M & 11,7 p. 100 & 96,5 p. 100 \\
Solution de présure commerciale & 7,8 p. 100 & 95,1 p. 100 \\
Eau distillée (présure commerciale dialysée) & & \\
\hline
\end{tabular}

\section{3) EFFET PROTECTEUR}

- Sérum albumine : après $4 \mathrm{~h}$ de couplage de la présure animale, $1 \mathrm{mg} / \mathrm{ml}$ de sérum albumine sont ajoutés au milieu; après $5 \mathrm{~h}$ de réaction, les billes de verre sont lavées et stockées. L'activité résiduelle atteint 38,3 p. 100 et le rendement 95,7 p. 100 . Par cette technique, l'activité résiduelle est augmentée de plus de 300 p. 100.

- Glutaraldéhyde : la sérum albumine a été remplacée par un agent bifonctionnel (la glutaraldéhyde). La durée d'action de la glutaraldéhyde est de 18 h. L'activité résiduelle est de 23,7 p.100 et le rendement de fixation de 97,1 p. 100.

\subsection{Amélioration de l'enzyme fixée au stockage}

Le tableau 2 reprend l'évolution de l'activité de l'enzyme fixée en fonction du temps et des différents milieux de conservation. 
TABLEAU 2

Activité de l'enzyme fixée (en p. 100 de l'activité initiale) lors du stockage

\begin{tabular}{|c|c|c|c|c|c|}
\hline \multirow{2}{*}{ Milieu de stockage } & \multicolumn{5}{|c|}{ Jours de stockage } \\
\hline & 1 & 3 & 6 & 9 & 13 \\
\hline Tampon $\mathrm{pH} 6,20,01 \mathrm{M}$ & 39,8 & 27,6 & 16,8 & 11,7 & 7,9 \\
\hline Tampon $\mathrm{pH} 7,00,01 \mathrm{M}$ & 72,7 & 38,5 & 10,2 & 8,1 & 7,6 \\
\hline Tampon $\mathrm{pH} 4,10,01 \mathrm{M}$ & 39,2 & 27,0 & 12,8 & 8,2 & 5,6 \\
\hline Tampon $\mathrm{pH} 4,8 \quad 0,01 \mathrm{M}$ & 42,7 & 31,7 & 18,8 & 15,6 & 13,9 \\
\hline Tampon pH 5,4 0,01 M & 50,1 & 34,7 & 25,1 & 17,6 & 13,1 \\
\hline $\mathrm{HCl} \mathrm{pH} 1,8$ & 49,3 & 37,1 & 22,5 & 14,8 & 10,9 \\
\hline Eau distillée & 81,0 & 42,1 & 34,1 & 22,5 & 17,5 \\
\hline $\mathrm{H}_{2} \mathrm{O}+\mathrm{NaCl} 0,1$ p. 100 & 59,0 & 36,0 & 22,3 & 12,6 & 6,6 \\
\hline
\end{tabular}

Quel que soit le milieu de conservation, l'activité de l'enzyme fixée décroît rapidement. Dans le meilleur des cas, 17,5 p.100 de l'activité initiale sont retrouvés après $13 \mathrm{j}$. La perte d'activité est due, pour une part importante à une dénaturation et non à un relâchement des protéines dans le milieu. Ainsi nous constatons que dans l'eau, 12,7 p. 100 de l'activité initiale est relâchée dans le milieu après $13 \mathrm{j}$; dans les tampons étudiés, le relâchement n'atteint pas 3 p. 100 de l'activité initiale. La stabilité de l'enzyme libre a été testée dans les différents milieux de conservation. Après $30 \mathrm{j}$ de conservation, l'enzyme libre garde entre 90 et 80 p. 100 de l'activité initiale. L'enzyme fixée sur billes séchées à $110^{\circ} \mathrm{C}$ après silanisation possède une meilleure stabilité au stockage (fig. 3). L'effet protecteur de la sérum albumine se marque au niveau de l'activité résiduelle, mais également lors de la conservation (fig. 3). Après 14 j, 42,2 p. 100 de l'activité initiale sont conservés.

Les facteurs étudiés aux paragraphes 3.2.1. et 3.2.2. n'ont aucun effet bénéfique sur la stabilité de l'enzyme immobilisée.

\section{IV. - DISCUSSION}

L'activité résiduelle de la présure immobilisée sur verre silanisé est faible (11,7 p. 100) malgré un rendement de fixation excellent 
(96,5 p. 100). Des résultats semblables ont été décrits dans la littérature $[5,1]$.

\subsection{Amélioration de l'activité résiduelle}

Les tentatives d'amélioration de l'activité résiduelle ont été réalisées à trois niveaux :

1) Etude de la préparation des billes de verre.

2) Etude du couplage.

3) Effet protecteur de certains produits.

1) ETUde De LA PRÉPARATION DES BILLES DE VERRE

Afin de renforcer l'efficacité de la silanisation, un chauffage des billes a été réalisé. Aucune amélioration de l'activité résiduelle n'a pu être mise en évidence.

Le remplacement de la réaction de diazotation par un agent bifonctionnel a également été testé. Cet agent permet la liaison entre le groupement $\mathrm{NH}_{2}$ du $\gamma$-aminopropyltriéthoxysilane et un $\mathrm{NH}_{2}$ de la protéine à fixer. Cette méthode nous a permis d'améliorer l'activité résiduelle de l'enzyme.

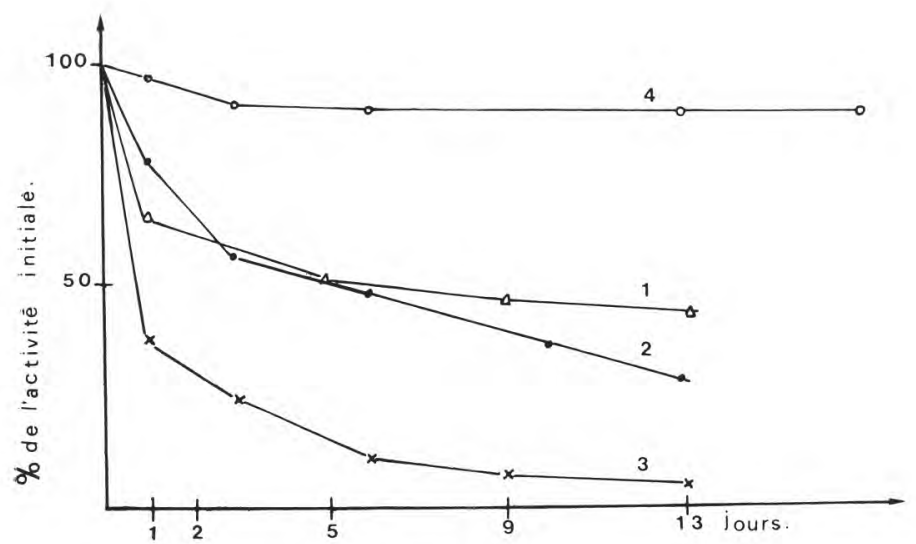

fig. 3

Etude de la stabilité de l'enzyme fixée en fonction du temps

(1) Influence de la protéine inerte (sérum albumine).

(2) Influence du séchage des billes après silanisation.

(3) Stabilité de l'enzyme fixée par la technique décrite dans les modes opératoires et conservée dans un tampon acétate $\mathrm{pH} \mathrm{4,8.}$

(4) Enzyme libre. 


\section{2) Etude du couplage}

La durée du couplage influence fortement le rendement de fixation (p. 100 des protéines fixées comparativement à la quantité de l'enzyme mise en œuvre) mais la proportion de protéines actives comparativement aux protéines fixées reste constante en fonction du temps de couplage. Le temps de ccuplage n'a donc pas d'influence sur l'activité résiduelle.

Le $\mathrm{pH}$ optimum de la réaction de couplage est voisin de 8 [7]. Malheureusement à ces $\mathrm{pH}$, l'enzyme est rapidement dénaturée. Il est nécessaire de trouver un compromis entre un bon rendement de réaction et des conditions de maintien de l'activité enzymatique. C'est pourquoi, nous avons utilisé des milieux de couplage de $\mathrm{pH}$ variant entre 6,2 et 7 . L'influence du $\mathrm{pH}$ et de la force ionique n'a pu être mise significativement en évidence au niveau de l'amélioration de l'activité résiduelle; dans nos conditions de travail, les rendements de fixation sont indépendants des facteurs étudiés.

\section{3) EFFET PROTECTEUR}

Après avoir immobilisé la présure, les sites activés inoccupés sont saturés par la sérum albumine (voir méthodes). L'activité résiduelle de la présure est fortement augmentée (38,3 p. 100 d'activité résiduelle) et le rendement de fixation reste constant. Le remplacement de la sérum albumine par un agent bifonctionnel permet également d'atteindre des activités résiduelles nettement plus élevées $(23,7$ p. 100$)$.

La glutaraldéhyde réagit sur deux groupements $\mathrm{NH}_{2}$ d'une même ou de différentes molécules d'enzyme. Le mode d'action des deux produits (sérum albumine et glutaraldéhyde) ne sont pas nécessairement comparables. La combinaison de l'action de la sérum albumine et de la glutaraldéhyde n'a pas permis d'améliorer les résultats obtenus par la seule addition de la sérum albumine. Signalons que l'effet protecteur de l'activité enzymatique par une protéine a également été signalée par Broun [2] et Dalhqvist [3].

\subsection{Amélioration de la stabilité de l'enzyme fixée au stockage}

La stabilité au stockage de l'enzyme fixée est faible quel que soit le milieu de conservation choisi (tab. 2). Il est significatif de remarquer que même à des $\mathrm{pH}$ acides $(4,8-5,2)$, aucune amélioration de stabilité n'a été enregistrée, alors que la présure libre présente une zone préférentielle de stabilité entre les pH 4,3 et 6,2.

Mickelsen [9] a présenté des résultats similaires au niveau de la stabilité de l'enzyme libre.

Arima [1] constate que 40 à 70 p. 100 des molécules actives de la présure fixée sur sépharose ou amino-éthyl cellulose sont relâchés 
dans le milieu après $4 \mathrm{j}$ de stockage. Green et Crutchfield [5] mettent également l'accent sur le relâchement de l'enzyme en fonction du $\mathrm{pH}$ et du support utilisés. Dans nos expériences sur verre poreux, le relâchement n'atteint jamais des valeurs aussi élevées, quel que soit le milieu de conservation. L'eau distillée est le milieu où le relâchement est le plus important mais l'activité résiduelle est légèrement supérieure aux autres milieux de conservation.

La stabilité de l'enzyme fixée a été testée dans tous les essais. Seuls l'addition de la sérum albumine et le séchage des billes après silanisation améliorent la stabilité de l'enzyme fixée (fig. 3).

Diverses causes peuvent être avancées pour expliquer l'effet protecteur de la sérum albumine sur la stabilité de l'enzyme fixée : forte concentration protéique qui stabiliserait la structure [2], diminution de la probabilité de coupler une même molécule à divers endroits au niveau du support, neutralisation des charges das sites inoccupés. Il est extrêmement difficile de donner une explication à l'effet positif du séchage des billes après silanisation sur la stabilité de l'enzyme fixée, à l'exception d'une augmentation de la force de liaison entre le produit silané et la bille poreuse.

\section{v. - CONCLUSIONS}

La méthode classique de fixation sur bille de verre par diazotation donne des résultats peu satisfaisants pour ce qui est de l'activité résiduelle et de la stabilité au stockage de la présure animale. L'activité résiduelle a pu être considérablement améliorée par la fixation d'une protéine inerte (la sérum albumine) ajoutée au milieu de couplage en fin de réaction et par l'addition d'un agent bifonctionnel (la glutaraldéhyde).

L'effet protecteur de la sérum albumine s'est également marqué sur la stabilité au stockage de l'enzyme fixée. Après 14 j, 42,2 p. 100 de l'activité initiale ont pu être conservés. Ces améliorations (activité résiduelle et stabilité au stockage) nous permettent d'envisager l'utilisation industrielle de la présure fixée.

L'application industrielle de la présure fixée est d'autant plus aisée que les problèmes sanitaires seront partiellement résolus par la possibilité de travailler à basse température. En effet, le coefficient de température $(Q$ 10) de la phase primaire de la coagulation du lait par la présure est égal à 2, alors que ce même paramètre est proche de 12 pour la phase secondaire.

\section{R és u mé}

L'activité résiduelle (de 11 à 40 p. 100) et la stabilité au stockage $(17,5$ à 42 p. 100) de la présure fixée par diazotation sur billes de 
verre poreux ont été considérablement améliorées. Différents facteurs intervenant au niveau de la réaction d'activation et de couplage, ainsi que certains agents jouant un rôle de protection de l'enzyme ont été étudiés.

\section{S u m m a r y}

Rennet was immobilized on porous glass. In the course of the study, we were able to get a 3 fold increase of the initial bound activity and a 2,5 fold increase of the half life of the immobilized enzyme. We essentially studied the activation reaction, the coupling to the substrate and various protecting agents.

\section{Remerciements}

Les auteurs remercient $M$. Coppens qui a suggéré le travail, M. Burny qui a participé à la rédaction de ce manuscrit ; le Comité d'Application des Méthodes Isotopiques et Radioactives en Agronomie est également remercié pour l'aide apportée au cours de ce travail.

Reçu pour publication en novembre 1975.

\section{Bibliographie}

[1] Arima (S.), Shimazaki (K), Yamazumi (T.), Kanamaru (Y.) (1974). - Japanëse Journal of Dairy Science, 23 (3), $\mathrm{A}_{83}-\mathrm{A}_{87}$.

[2] Broun (G.), Thomas (D.), Gellf (G.), Domurado (D.), Berjonneau (A. M.), Guillon (G.) (1973). - Biotechn. Bioeng., 15, 359.

[3] Dahlovist (A.), Mattiasson (B.), Mosbach (K.) (1973). - Biotechn. Bioeng., 15,395 .

[4] Durand (G.), Baudras (A.) (1975). - La Recherche, 56, 437.

[5] Green (M. L.), Crutchfield (G.) (1969). - Biochem. J., 115, 183.

[6] Houins (G), Deroanne (C), Coppens (R.) (1973). - Le Lait, 529-530, 610.

[7] Messing (R. A.), Weetall (H. H.) (1970). - U.S. Patent, 3, 519.

[8] Messing. - Immobilized Enzymes for Industrial Reactors 1975. Academic Press New York-London.

[9] Mickelsen (R.), Ernstrom (C. A.) (1967). - J. Dairy Sci., 50 (5), 645.

[10] Stanley (W.) Olson (A.) (1974). - J. Food Sci., 39, 660.

[11] Weetall (H. H.) (1969) -. Science, 166, 615.

[12] Woychiк (J. H.), Wondolowski (M. V.) (1972). - Biochim. Biophys. Acta, $289,347$.

[13] ZABORSKY (O. R.) (1973). - Immobilized Enzymes. CRC Press Cleveland (Ohio - U.S.A.). 